\title{
A woman with fever and cough: coronavirus disease 2019
}

\author{
Yuan-Qiang $\mathrm{Lu}^{1}$
}

Received: 10 March 2020 / Accepted: 20 March 2020 / Published online: 30 March 2020

(c) Società Italiana di Medicina Interna (SIMI) 2020

A 64-year-old woman presented to the emergency department on February 1st, 2020, because of a 7-day fever, with the highest body temperature of $38.7^{\circ} \mathrm{C}$, accompanied by cough with a little sputum and muscle soreness, but no chest distress or dyspnea. She just flew back to Hangzhou, China, from Malaysia 7 days ago. Physical examination revealed no dry and moist rales in both lungs with a temperature of $37.3{ }^{\circ} \mathrm{C}$, heart rate of 92 beats/min, respiratory rate of 18 times/min, and blood pressure of $113 / 79 \mathrm{mmHg}$. Her laboratory tests showed white blood cell count (WBC) was $2.5 \times 10^{9}$ cells $/ \mathrm{mL}$ with $63.3 \%$ neutrophils and $29.2 \%$ lymphocytes, and C-reactive protein (CRP) level was $3.62 \mathrm{mg} / \mathrm{L}$. Her sputum test for influenza A $+B$ (real-time fluorescent RT-PCR) was negative, and the lung CT images were shown in Fig. 1. The lung CT images of this patient showed multiple ground-glass shadows with unclear edges and uneven densities in the peripheral and posterior lungs, mainly under the pleura. These imagings were in accordance with the early radiographic features of coronavirus disease 2019 (COVID19). Later, the severe acute respiratory syndrome coronavirus 2 (SARS-Cov-2) RNA was detected in the sputum by real-time fluorescent RT-PCR, and the patient was diagnosed as COVID-19.

A pneumonia of unknown etiology has spread rapidly all over the world since December 2019 [1]. In January 2020, the new virus was successfully isolated and sequenced [2], and then, the virus and corresponding disease were formally named the SARS-Cov-2 and COVID-19 by the World Health
Organization. The COVID-19 has the characteristics of strong transmissibility, long disease course, and rapid exacerbation. This virus belongs to the Beta coronavirus genus, and its pathogenic mechanism has not been clarified, which need to be further studied.

The diagnosis of pulmonary diseases mainly depends on the examination of etiology, and SARS-Cov-2 RNA detection is the gold standard for the diagnosis of COVID-19. Clinically, the lung CT imaging of patients with COVID-19 has unique characteristics [3], which show multiple groundglass shadows and interstitial changes, mainly in the peripheral lungs at the early stage, and then multiple opaque infiltrates and pulmonary consolidations in both lungs, with rare pleural effusions. In this case, the patient was treated with patient isolation, antiviral drugs, small doses of glucocorticoids, and high-flow nasal oxygen therapy immediately. Then, she recovered uneventfully and was discharged on day 16.
Yuan-Qiang Lu

luyuanqiang@zju.edu.cn

1 Department of Emergency Medicine, Zhejiang Provincial Key Laboratory for Diagnosis and Treatment of Aging and Physic-Chemical Injury Diseases, The First Affiliated Hospital, School of Medicine, Zhejiang University, Hangzhou 310003, Zhejiang, People's Republic of China 

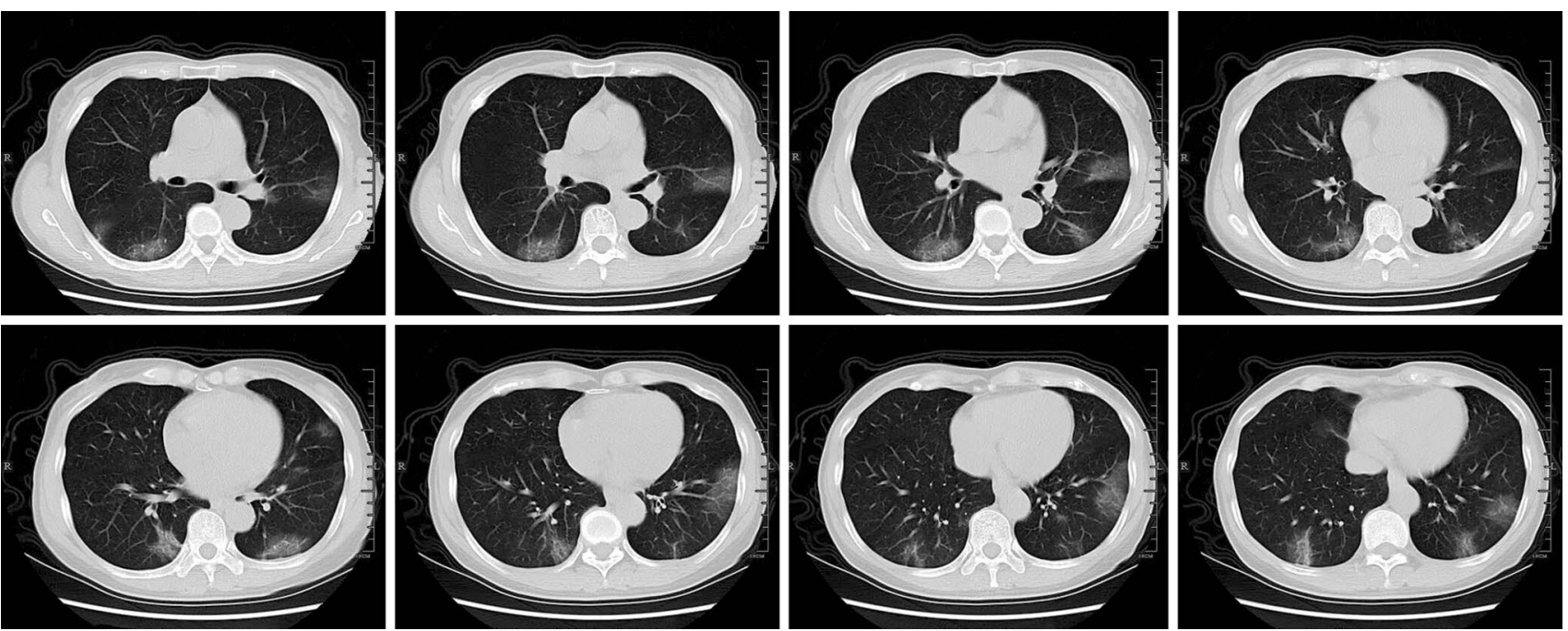

Fig. 1 Lung CT images of this patient showed multiple ground-glass shadows with unclear edges and uneven densities in the peripheral and posterior lungs, mainly under the pleura

Funding This study was sponsored by the Foundation of Key Discipline Construction of Zhejiang Province for Traditional Chinese Medicine (no. 2017-XK-A36).

\section{Compliance with ethical standards}

Conflict of interest The authors declare that they have no conflict of interest.

Ethical standards The manuscript does not contain clinical studies or identifying patient data.

Statement of human and animal rights This article does not contain any studies with human participants or animals performed by any of the authors.

Informed consent Given the absence of identifying patient data, informed consent was deferred.

\section{References}

1. Zhu N, Zhang D, Wang W et al (2019) A Novel Coronavirus from Patients with Pneumonia in China. N Engl J Med 382:727-733

2. Chen L, Liu W, Zhang Q et al (2020) RNA based mNGS approach identifies a novel human coronavirus from two individual pneumonia cases in 2019 Wuhan outbreak. Emerg Microbes Infect 9:313-319

3. Song F, Shi N, Shan F et al (2020) Emerging coronavirus 2019nCoV pneumonia. Radiology (published online ahead of print, 2020 Feb 6)

Publisher's Note Springer Nature remains neutral with regard to jurisdictional claims in published maps and institutional affiliations. 